\title{
Differentiation Therapy with Cancer Stem Cells
}

\section{Lin $\mathrm{CY}^{*}$}

Departments of Neurosurgery, Orthopaedic Surgery, and Biomedical Engineering, University of Michigan, USA

Recent studies have shown that many tumors contain a small population of cells, termed tumor-initiating cells or cancer stem cells (CSCs), that are responsible for tumor progression, metastasis, and resistance to chemotherapy and radiation treatment. This new CSC model has given a new perspective on the origin of cancer and the development of the heterogeneous hierarchy that constitutes tumor populations. Moreover, this finding has shifted an old idea to a novel paradigm that inspires new strategies to fight cancer. For example, increased attention has focused on targeting specific cell surface markers (antigens) contained in these stem cells. So far, the most extensively studied antigen is CD133. New drugs for the treatment of CSCs have also been designed to target cellular mechanisms that regulate cell proliferation, including Bmi-1, Notch, Sonic hedgehog and Wnt signaling pathways. Nonetheless, these attempts are generally quite tissue-specific, making it difficult to extensively target CSCs across tumors to achieve broad therapeutic efficacy.

The origin of CSCs and whether they are attributed to tumor initiation are still controversial. Advocators of the cancer stem cell hypothesis postulate that most human cancers derive from a single cell targeted by genetic and epigenetic alterations that initiate malignant transformation, and progressively, these early cancer cells give rise to different generations of daughter cells that accumulate additional mutations, acting in concert to drive the full neoplastic phenotype. This phenomenon has been observed in certain tissue-specific stem cells. The strongest evidence to support this hypothesis is dysregulated hematopoietic stem cells (HSCs) that lead to leukemogenesis. Cells that have a phenotype CD34+CD38- similar to normal HSCs have been found capable of initiating human acute myeloid leukemia (AML) and most of the subtypes [1,2]. The same paradigm also applies to several other malignant tissues, including brain, breast, and pancreatic cancers. However, examples exist that apparently contradict the cancer stem cell theory being a general rule. A report indicated that at least certain malignancies, particularly those with substantial homogeneity, such as lymphomas or leukemias, can be maintained by the majority of the original population instead of the "side-population" of CSCs [3]. Another report even showed the evidence that a human glioma was overwhelmingly initiated by each of the selected single cancer cells plated in culture, many of which are exclusive of stem cell features [4].

Perhaps the model proposed by Stewart Sell may give a more thorough picture of CSCs in cancer initiation and promotion [5]. The idea is that the most primitive stem cells normally do not divide in adult tissues, and there is asymmetric division of transit-amplifying cells, so that one daughter cell remains a progenitor cell whereas the other differentiates. Cancer develops when there is a block during the maturation scheme, thus termed maturation arrest, resulting in retention of the property of symmetric division in both daughter cells. The maturation arrest should occur in self-renewing progenitor cells, for they do not undergo typical turnover like mature cells and remain present before proliferative stimulus is given. The concept of this model also explains the differentiation state of the cancer that develops will depend on the stage of differentiation at which maturation arrest occurs. To this point, differentiation therapy has been proposed to induce terminal differentiation of the cancer stem or progenitor cells so that active proliferation and chemoresistance can be suppressed. The therapy has been encouraged by some preclinical and clinical outcomes including inhibiting glioblastoma expansion with BMP-4 [6], and in treating acute promyelocytic leukemia with retinoic acids (vitamin A analogs) to remove the maturation block [5]. The proposed study is aimed at preliminarily validating the concept described above. Our recent reports also demonstrated that the malignant potential of osteosarcoma is reflective of the level of retained stem cell attributes and aldehyde dehydrogenase (ALDH) enzymatic activity $[7,8]$. When these putative CSCs were exposed to BMP-2, they were induced to express osteogenic phenotypes without stimulating proliferation, thereby drastically attenuating tumor formation and expansion. Moreover, the heterogenous progenies associated with low ALDH expression were induced to generate structured, calcified bone tissue in vivo [9]. Recently, inhibition of aldehyde dehydrogenase (ALDH) activity was shown to reduce chemotherapy and radiation resistance of stem-like ALDHhiCD44+ human breast cancer cells through their differentiation [10].

Elimination or interference with the 'stemness' of CSCs by triggering epigenetic alterations may shed insight into developing a new strategy to control tumor propagation. The current evaluations have provided preliminary evidence of induced differentiation to effectively deplete tumorigenesis by the tumor initiating cells. Such pilot work has also formed a foundation for pursuing further studies into the mechanisms of underlying interactions between exogenous growth factors and CSCs.

\section{References}

1. Bonnet D, Dick JE (1997) Human acute myeloid leukemia is organized as a hierarchy that originates from a primitive hematopoietic cell. Nat Med 3: 730737

2. Reya T, Morrison SJ, Clarke MF, Weissman IL (2001) Stem cells, cancer, and cancer stem cells. Nature 414: 105-111.

3. Kelly PN, Dakic A, Adams JM, Nutt SL, Strasser A (2007) Tumor growth need not be driven by rare cancer stem cells. Science 317: 337-337.

4. Zheng X, Shen G, Yang X, Liu W (2007) Most C6 cells are cancer stem cells: evidence from clonal and population analyses (vol 67, pg 3691, 2007). Cancer Res 67: 10097-10097.

5. Sell S (2006) Cancer stem cells and differentiation therapy. Tumour Biology 27: $59-70$.

6. Piccirillo SGM, Reynolds BA, Zanetti N, Lamorte G, Binda E, et al. (2006) Bone morphogenetic proteins inhibit the tumorigenic potential of human brain tumour-initiating cells. Nature 444: 761-765.

*Corresponding author: Chia-Ying Lin, Ph.D., Department of Neurosurgery University of Michigan, 109 Zina Pitcher Place BSRB 5007, Ann Arbor, Michigan 48109, USA, Tel: +17346150371; E-mail: lincy@umich.edu

Received January 14, 2014; Accepted January 17, 2014; Published January 20 2014

Citation: Lin CY (2014) Differentiation Therapy with Cancer Stem Cells. J Stem Cell Res Ther 4: e117. doi:10.4172/2157-7633.1000e117

Copyright: (c) 2014 Lin CY. This is an open-access article distributed under the terms of the Creative Commons Attribution License, which permits unrestricted use, distribution, and reproduction in any medium, provided the original author and source are credited. 
7. Wang L, Park P, Lin CY (2009) Characterization of Stem Cell Attributes in Human Osteosarcoma Cell Lines. Cancer Biol Ther 8: 543-552.

8. Wang, L, Park P, Zhang H, La Marca F, Lin CY (2011) Prospective identification of tumorigenic osteosarcoma cancer stem cells in OS99-1 cells based on high aldehyde dehydrogenase activity. International journal of cancer. Int J Cancer 128: 294-303.
9. Wang L, Park P, Zhang HN, La Marca F, Claeson A, et al. (2011) BMP-2 inhibits the tumorigenicity of cancer stem cells in human osteosarcoma OS99-1 cell line. Cancer Biol Ther 11: 457-463.

10. Croker AK, Allan AL (2012) Inhibition of aldehyde dehydrogenase (ALDH) activity reduces chemotherapy and radiation resistance of stem-like ALDHhiCD44(+) human breast cancer cells. Breast Cancer Res Treat 133: 75-87. 